\title{
A Rotationally Symmetric Lateral Distribution Function for Radio Emission from Inclined Air Showers
}

\author{
Tim Huege ${ }^{1,2, \star}$, Lukas Brenk ${ }^{1}$, and Felix Schlüter ${ }^{1}$ \\ ${ }^{1}$ Karlsruhe Institute of Technology, Institute for Nuclear Physics, Karlsruhe, Germany \\ ${ }^{2}$ Vrije Universiteit Brussel, Brussels, Belgium
}

\begin{abstract}
Radio detection of inclined air showers is currently receiving great attention. To exploit the potential, a suitable event reconstruction needs to be developed. A crucial step in this direction is the development of a model for the lateral distribution of the radio signals, which in the case of inclined air showers exhibits asymmetries due to "earlylate" effects in addition to the usual asymmetries from the superposition of charge-excess and geomagnetic emission. We present a model which corrects for all asymmetries and successfully describes the lateral distribution of the energy fluence with a rotationally symmetric function. This gives access to the radiation energy as a measure of the energy of the cosmic-ray primary, and is also sensitive to the depth of the shower maximum.
\end{abstract}

\section{Introduction}

Due to the superposition of geomagnetic and charge-excess emission, the distribution of the energy fluence of the radio emission from extensive air showers is asymmetric on the ground [1]. Lateral distribution functions (LDFs) have to take into account this asymmetry by either a two-dimensional description [2], by correcting for the asymmetry induced by the charge-excess contribution [3], or by treating the two contributions individually [4]. In case of inclined air showers, additional asymmetries arise from "early-late effects", i.e., the fact that the emission above the shower axis propagates longer through the atmosphere than the emission below the shower axis.

In this article, we first present methods to correct for the early-late asymmetry as well as the charge-excess-induced asymmetry in the energy-fluence footprints of inclined air showers. We then propose a rotationally symmetric LDF that, when fit to the symmetrized energy fluences, allows precise determination of the cosmic-ray energy for inclined air showers.

\section{Correction of early-late effects}

Asymmetries introduced by early-late effects can be easily corrected to first order by assuming that the emission originates from a point source situated at the shower maximum, at geometrical distance $R_{0}$ from the core. Axis distances of antennas $r_{\text {raw }}$ need to be projected along the line of sight from antenna to source into the shower plane containing the core, resulting in the modified lateral distance $r$ as illustrated in Fig. (1). This is equivalent to describing the radiation pattern in terms of off-axis angles rather than axis distances.

^e-mail: tim.huege@kit.edu 


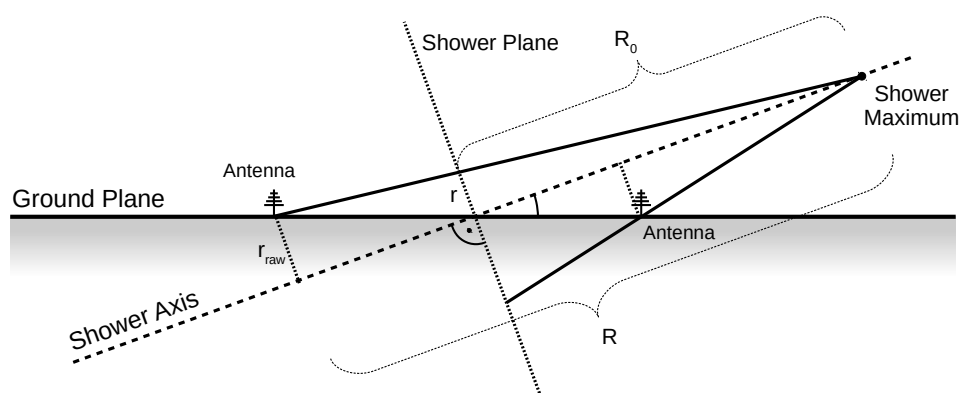

Figure 1. Illustration of the correction for early-late effects.

The energy fluences $f_{\text {raw }}$ measured in the antennas need to be corrected for the corresponding change of the distance between antenna and source using an inverse square law. (Amplitudes would require an inverse linear correction.) In summary:

$$
f=f_{\text {raw }} \cdot\left(\frac{R}{R_{0}}\right)^{2} \quad r=r_{\text {raw }} \cdot \frac{R_{0}}{R}
$$

With the application of these two corrections, early-late asymmetries are effectively removed, possibly allowing the successful application of other approaches such as the one presented in [4] to inclined air showers.

\section{Charge-excess correction}

After application of the early-late correction, the contribution of the charge-excess radiation needs to be deducted from the total energy fluences $f$ to determine the "geomagnetic energy fluences" $f_{\text {geo }}$. This can be done in two ways.

We have derived a parameterization for the charge-excess fraction $a=\sin ^{2}(\alpha) f_{\mathrm{CE}} / f_{\text {geo }}$ as a function of zenith angle $\theta$, axis distance $r$, and depth of shower maximum $X_{\max }$ :

$$
a\left(\theta, r, X_{\max }\right)=\cos (\theta)^{4.118} \cdot\left(\frac{r}{7937 \mathrm{~m}}\right) \cdot \exp \left(\frac{r}{1221 \mathrm{~m}}\right) \cdot \exp \left(\frac{X_{\max }}{331.5 \mathrm{~g} / \mathrm{cm}^{2}}\right) .
$$

This parameterization for the frequency band from 30 to $80 \mathrm{MHz}$ has been determined from CoREAS [5] simulations of air showers in the zenith-angle range from 60 to $80^{\circ}$ spanning energies from 4 to $40 \mathrm{EeV}$ for an antenna-array altitude, average atmosphere, and magnetic field configuration as valid for the site of the Auger Engineering Radio Array [6]. For other locations, the numerical parameters need to be re-determined.

Using the known polarization characteristics of the charge-excess and geomagnetic components, and assuming that both follow a rotationally symmetric LDF with negligible circular polarization [7], the geomagnetic energy fluence can then be determined from a measurement of the energy fluence in the polarization defined by the direction of the Lorentz force $\mathbf{v} \times \mathbf{B}$ as:

$$
f_{\text {geo }}(r)=\frac{f_{\mathbf{v} \times \mathbf{B}}(r)}{\left(1+\cos (\phi) \cdot \sqrt{a\left(\theta, r, X_{\max }\right)} / \sin \alpha\right)^{2}},
$$

where $\phi$ denotes the polar angle equalling $0^{\circ}$ in the direction given by the Lorentz force and counting counter-clockwise. 

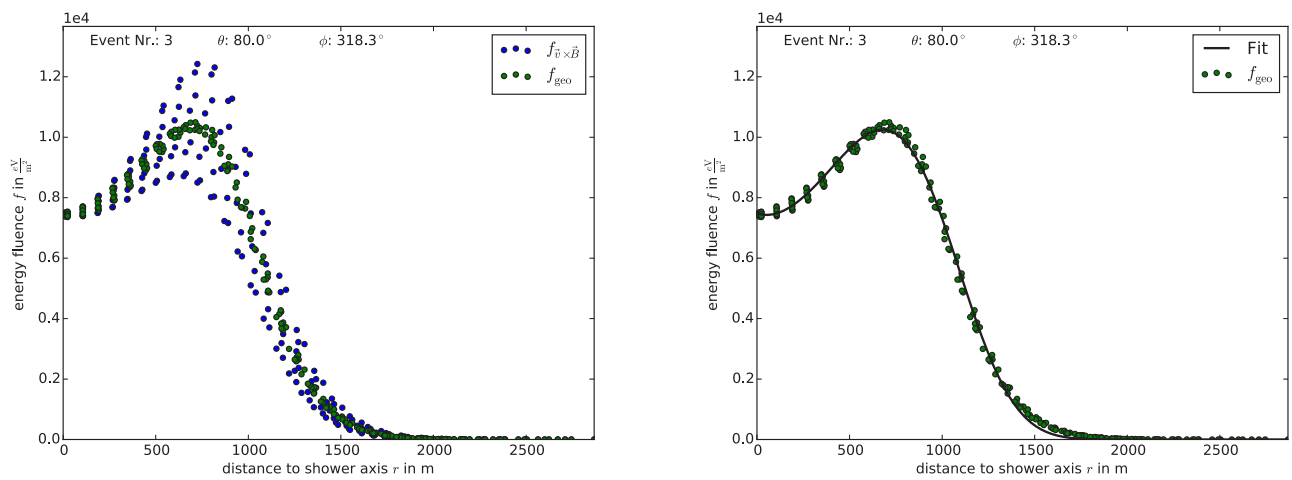

Figure 2. Left: Distribution of energy fluences before (blue) and after (green) symmetrization through deduction of the charge-excess component. Right: Illustration of the LDF fit with equation Eq. (4).

Alternatively, in particular if the signal-to-noise ratio of measurements in the polarization component perpendicular to the Lorentz force is good, the charge-excess fraction can be determined from the electric-field polarization of the signal measured at each individual antenna directly, without reverting to the above parameterization.

\section{Symmetrical lateral distribution function}

Now that the signal distributions have been symmetrized (see Fig. (2) left), a one-dimensional LDF can be readily fit to the energy fluences as a function of lateral distance $r$ (see Fig. (2) right). We use an exponential of a cubic polynomial as fit function. This is a generalization of the exponential of a quadratic function previously used by Tunka-Rex to fit measured amplitudes [3].

$$
f_{\text {geo }}^{\text {fit }}(r)=A \exp \left(-B r-C r^{2}-D r^{3}\right)
$$

\section{Determination of the radiation energy}

After the one-dimensional fit has been applied, an area-integration can easily be performed to determine the radiation energy. Given that the charge-excess contributions to the energy fluences have been removed, this yields the radiation energy of the geomagnetic emission. We verified that, as expected, it scales quadratically with the cosmic-ray energy. It exhibits a spread of $10 \%$. Correlation with the energy in the electromagnetic cascade rather than the cosmic-ray energy, and application of further corrections on the air density at shower maximum [7] are bound to further improve this resolution.

\section{References}

[1] T. Huege, Physics Reports 620, 1 (2016)

[2] A. Nelles, S. Buitink, H. Falcke, et al., Astropart. Phys. 60, 13 (2015)

[3] D. Kostunin, P.A. Bezyazeekov, R. Hiller, et al., Astropart. Phys. 74, 79 (2015)

[4] C. Glaser, S. de Jong, M. Erdmann, J.R. Hörandel, Astropart. Phys. 104, 64 (2019)

[5] T. Huege, M. Ludwig, C.W. James, AIP Conf. Proc. 1535, 128 (2013)

[6] J. Rautenberg for the Pierre Auger Collaboration, these proceedings

[7] C. Glaser, M. Erdmann, J.R. Hörandel, T. Huege, J. Schulz, JCAP 09, 024 (2016) 